\title{
Sulfato de magnesio y protección neuronal prenatal
}

\author{
Álvaro Monterrosa-Castro. ${ }^{1}$, Ivette Romero-Pérez. ${ }^{1}$, Elida Caraballo-Olave. ${ }^{2}$ \\ ${ }^{1}$ Grupo de Investigación Salud de la Mujer, ${ }^{2}$ Miembro del semillero de investigación FEM-SALUD del Grupo de \\ Investigación Salud de la Mujer. Facultad de Medicina, Universidad de Cartagena. Cartagena, Colombia.
}

\section{RESUMEN}

Antecedentes: La prematuridad es importante factor de riesgo para el desarrollo de parálisis cerebral (PC). El Sulfato de Magnesio (MgSO4) se ha planteado como una estrategia para reducir el riesgo de PC en recién nacidos por debajo de las 34 semanas de gestación. Objetivo: Precisar con la evidencia disponible, la validez del uso del MgSO4 para protección neuronal prenatal en embarazadas en riesgo de parto pretérmino (PP) inminente. Método: Se revisaron las bases de datos PubMed, ScienceDirect, EBSCOhost, Scielo y OvidSP en búsqueda de estudios clínicos y epidemiológicos, revisiones sistemáticas, consensos y meta análisis. Se realizó revisión temática de los artículos que cumplieron los criterios de selección. Resultados: Experimentos en modelos animales mostraron la posibilidad que el MgSO4 fuese protector neuronal. Estudios observacionales señalaron la posible asociación entre la exposición fetal al MgS04 y reducción en morbilidad neurológica en nacidos pretérmino (NP). Cinco ensayos clínicos entre 2002-2008, individualmente no mostraron datos concluyentes. En el 2009 se publicaron tres metaanálisis, basados en esos mismos ensayos y mostraron significativa reducción de PC en NP expuestos prenatalmente al MgSO4. Conclusión: Existe evidencia para recomendar MgSO4 para protección neuronal prenatal antes de las 34 semanas de embarazo y con riesgo inminente de PP, aunque no está definida la dosis óptima. Se recomienda aplicar hasta el parto o por 12-24 horas.

\section{PALABRAS CLAVE: Parálisis cerebral, sulfato de magnesio, parto prematuro}

\section{SUMMARY}

Background: Prematurity is a leading risk factor for development of cerebral palsy (CP). The use of Magnesium sulphate (MgSO4) has been proposed as a strategy to reduce the risk of cerebral palsy in preterm infants less than 34 weeks of gestation. Aims: To assess the best available evidence in order to validate the use of $\mathrm{MgSO} 4$ for prenatal neuroprotection in pregnant women at risk of imminent preterm delivery. Methods: we searched the PubMed, ScienceDirect, EBSCOhost, Scielo and OvidSP databases for clinical and epidemiological studies, systematic reviews, consensus and meta-analysis about the use of Magnesium sulphate to prevent cerebral palsy. Thematic review was conducted of articles that met the selection criteria. Results: Experiments in animal models showed properties of MgSO4 for neuroprotection. Observational studies indicated the possible association between fetal exposures to MgS04 and reduced neurological morbidity in PP. Five clinical trials between 2002 and 2008 showed no conclusive data individually. In 2009, three meta-analysis showed significant reduction of cerebral palsy in MgSO4 exposed preterm infants. Conclusion: There is evidence to recommend the use of $\mathrm{MgSO} 4$ for prenatal neuroprotection before 34 weeks of pregnancy and imminent risk of preterm birth. It is unclear the optimal dose of MgSO4; is recommended until delivery or by 12-24 hours.

KEY WORDS: Cerebral palsy, magnesium sulfate, premature labor 


\section{INTRODUCCIÓN}

Los avances en los cuidados sanitarios de los nacidos pretérminos (NP) han traído como resultado el incremento en la tasa de supervivencia (1). Sin embargo, también ha aumentado la prevalencia de discapacidades neurosensoriales asociadas con la prematurez como: parálisis cerebral (PC), limitaciones visuales y auditivas, retrasos del desarrollo, dificultad en el aprendizaje, problemas psicológicos y de comportamiento (2).

Como PC se describe un grupo de trastornos permanentes del desarrollo del movimiento y de la postura, que causan limitaciones en la actividad y atribuidos a alteraciones no progresivas ocurridas en el desarrollo cerebral del feto o del lactante. Los trastornos motores de la PC están a menudo acompañados por alteraciones de la sensación, percepción, cognición, comunicación y conducta, y/o por trastornos convulsivos (2).

La prematuridad es un importante factor de riesgo para el desarrollo de PC $(3,4)$. El riesgo de PC es casi 80 veces mayor en nacidos de menos de 28 semanas de gestación que en nacidos a término (RR: 78,9; IC95\% 56,5-110,0) (4). La prevalencia global de PC se estima en 1 por cada 276 nacidos (5). Los costos de atención de por vida para los pacientes con PC es sensiblemente alto si se considera la atención médica directa, costos directos no médicos (modificaciones en el hogar y automóvil, educación especial) e indirectos como la pérdida en la productividad. El Centro de Control y Prevención de Enfermedades (CDC) de Estados Unidos calculó en 11,5 billones de dólares el costo para todos los nacidos con PC en el año 2000. Para el año 2003, el costo estimado de atención de la PC fue de un millón de dólares por cada caso (6).

Experimentos en modelos animales mostraron la posibilidad que el MgSO4 fuese protector neuronal, mientras que estudios observacionales señalaron la posible asociación entre la exposición fetal al MgS04 y la reducción en morbilidad neurológica en nacidos pretérmino.

El objetivo de este estudio es precisar con la evidencia disponible, la validez del MgSO4 para protección neuronal prenatal, en embarazadas en riesgo de parto pretérmino (PP) inminente.

\section{METODOLOGÍA}

Tipo de estudio: revisión temática. Se incluyeron en la búsqueda estudios clínicos y epidemiológicos, revisiones sistemáticas, temáticas, consensos, reuniones de expertos, metaanálisis y guías clínicas o protocolos.

Tipo de participantes: artículos publicados sobre eficacia y efectividad, dosis y protocolos de administración del MgSO4 para protección neuronal prenatal en mujeres en riesgo de PP inminente, definido como trabajo de parto activo antes de las 37 semanas de gestación, cuatro o más centímetros de dilatación cervical y con o sin ruptura de membranas. También se consideró PP planeado, como la interrupción del embarazo antes de las 37 semanas por indicaciones fetales o maternas.

Estrategia de búsqueda bibliográfica: búsqueda electrónica en las bases de datos PubMed, ScienceDirect, EBSCOhost, OvidSP y Scielo, entre enero del 2000 y noviembre del 2012. Las lenguas consideradas fueron inglés y español.

Términos claves: se realizó la búsqueda con las palabras claves obtenidas del Mesh: cerebral palsy; magnesium sulfate; premature birth; infant premature; infant premature diseases; obstetric labor premature. También con las palabras claves obtenidas del DeCS: parálisis cerebral; sulfato de magnesio; nacimiento prematuro; prematuro; enfermedades del prematuro; trabajo de parto prematuro.

Métodos de revisión: se realizó búsqueda de artículos identificando la pertinencia de los títulos. Se eliminaron los repetidos. Se revisaron los resúmenes de forma separada e independiente. Se escogieron los resúmenes de artículos y de publicaciones en cualquier modalidad que presentara información que se ajustaran al objetivo propuesto. Seguidamente se adquirieron las publicaciones en texto completo. Solo se consideraron artículos que estuvieran totalmente disponibles.

Recopilación y análisis de datos: se evaluaron los artículos de forma independiente.

Criterios de selección: se incluyeron estudios sin restricciones de diseño ni nivel de evidencia.

\section{RESULTADOS}

Se identificaron 230 títulos, de ellos se consideraron $56(24 \%)$ resúmenes como pertinentes y todos fueron adquiridos en texto completo.

MgSO4 y protección neuronal prenatal: Se denomina protección neuronal a aquellas medidas que tomadas concomitante o antes de un evento hipóxico o isquémico, incrementan la tolerancia neuronal y mejoran su supervivencia (7).

Desde los años ochenta y noventa se había observado en modelos animales con lesiones cerebrales adquiridas, que el MgSO4 reducía el tamaño de lesiones producto de hipoxia y daño isquémico, prevenía la apoptosis, conservaba la función de la membrana celular neuronal, entre otros, lo que permitía considerar la posibilidad de protección neuronal (8).

A finales de 1980 , dos estudios $(9,10)$ señalaron que NP de mujeres con preeclampsia, tenían menor incidencia de hemorragia en el sistema nervioso central, que nacidos de madres sin preeclampsia con igual edad gestacional. 
En un estudio de casos y controles, Nelson y Grether (11) en 1995, se preguntaron si la administración materna de MgSO4 reducía el riesgo de PC en recién nacidos con peso inferior a 1500 gramos. Fue el primer estudio observacional que señaló que la exposición fetal a MgSO4 podía estar asociada a reducción en el riesgo del desarrollo subsecuente de PC en NP (OR: 0,14; IC95\% 0,05-0,51).

En los años siguientes otros estudios observacionales apoyaron o refutaron el hallazgo. El MgSO4 fue administrado a las mujeres involucradas en esos estudios por sus propiedades tocolíticas o para la prevención de convulsiones en mujeres con preeclampsia/eclampsia. Cinco ensayos clínicos se publicaron entre los años 2002-2008 (12-17) evaluando el uso de MgSO4 para protección neuronal prenatal, específicamente PC, en mujeres en riesgo de PP inminente o PP programado.

El primero fue el estudio MagNET (Magnesium and Neurologic Endpoints Trial) adelantado en un centro norteamericano y realizado en 149 mujeres en trabajo de PP entre 25 y 33 semanas de gestación, incluyendo embarazos únicos y múltiples; 92 embarazadas tenían menos de 4 centímetros de dilatación y fueron elegidas para tocolisis. La mitad recibió MgSO4 y la otra mitad otros agentes; 57 mujeres tenían más de 4 centímetros de dilatación, a 29 se les administró MgSO4 con fines de "protección neuronal" y solución salina a las otras 28 mujeres. El estudio fue detenido tempranamente debido a una alta tasa de mortalidad pediátrica en el grupo de MgSO4; el estudio permitió observar tasa de PC en 3/85 infantes que fueron expuestos in-útero a MgSO4 y en $3 / 80$ no expuestos (RR: 0,94; IC95\% 0,20-4,53). El estudio no soportó el efecto benéfico en protección neuronal que anteriormente había sido propuesto (12).

En 2003 se publicó el estudio ACTOMgSO4 (Australasian Collaborative Trial Of MagnesiumSulphate) (13), realizado en 1062 mujeres con embarazo único o múltiple de menos de 30 semanas de gestación, que tuvieron parto antes de 24 horas de ingreso a 16 centros hospitalarios de Australia y Nueva Zelandia; 535 mujeres y sus 629 fetos vivos fueron expuestos a MgSO4 y 527 mujeres y sus 626 fetos vivos a solución salina. A diferencia del estudio previo, no se presentó elevación en la tasa de mortalidad perinatal, 87/629 en los expuestos y 107/626 en los no expuestos (RR: 0,83; IC95\% $0,64-1,09)$. Los infantes fueron valorados clínicamente a los dos años y la función motora evaluada por GMFCS (Gross Motor Functions Classification System). La tasa de PC fue similar en ambos grupos 36/629 en expuestos y 42/626 en no expuestos (RR: 0,83; IC95\% 0,54-1,27). La tasa de disfunción motora sustancial estuvo significativamente reducida (RR: 0,51; IC95\% 0,29-0,91), en expuestos $18 / 629$, frente a $34 / 626$ en no expuestos. Disfunción motora sustancial fue definida como la inha- bilidad para caminar sin asistencia. El MgSO4 administrado inmediatamente antes del PP podía ofrecer protección neuronal antenatal.

En el año 2007 se publicó un primer informe del estudio PREMAG (14), realizado en 564 mujeres que ingresaron a 18 centros hospitalarios en Francia con embarazo único o múltiple de menos de 33 semanas de gestación y tuvieron parto antes de 24 horas; 286 mujeres recibieron una dosis única de 4 gramos de MgSO4 y 278 de solución salina, 354 fetos fueron expuestos y 341 no expuestos.

Se encontró que la mortalidad pediátrica era similar en los dos grupos, 33/352 en los expuestos y 35/336 en los no expuestos (OR: 0,79; IC95\% 0,44$1,44)$. Todos los recién nacidos fueron valorados en las primeras semanas, con ultrasonido craneal en búsqueda de injurias en la sustancia blanca. Formas severas de injuria en la sustancia blanca fueron encontradas en 34/352 de los expuestos y en 38/336 de los no expuestos (OR: 0,78; IC95\% 0,47-1,31). En la evaluación conjunta de injuria severa de la sustancia blanca y/o muerte tampoco se encontró diferencia significativa (OR: 0,86; IC95\% 0,55-1,34). Si bien se encontraron porcentualmente menos lesiones en expuestos, las diferencias no fueron significativas en la evaluación de injurias en la sustancia blanca de todas las magnitudes (OR: 0,79; IC95\% 0,51-1,22), quistes periventriculares (OR: 0,94; IC95\% 0,531,68 ), hemorragias intraparenquimatosas (OR: 0,42; IC95\% 0,14-1,21), hemorragias no parenquimatosas (OR: 0,75, IC95\% 0,50-1,11), hemorragias subependimal (OR: 0,77;IC95\% 0,49-1,21), hemorragia intraventricular aislada (OR: 0,87; IC95\% 0,47-1,62) y hemorragia intraventricular con dilatación (OR: 0,76; IC95\% 0,31-1,84). Los autores señalaron que la falta de significancia podía guardar relación con la elevada cantidad de gestantes con ruptura prematura de membranas e infección materno-fetal. También consideraron que la sincronización entre la dosis de infusión y la cascada de eventos que llevan a la injuria de la sustancia blanca pudo ser inapropiado, la dosis inicial muy baja o no ser lo adecuado la ausencia de infusión de mantenimiento.

Un año más tarde se publicó un nuevo informe del PREMAG valorando morbilidad neurológica luego de dos años de seguimiento (15). La valoración fue realizada con un cuestionario para valorar desarrollo cognitivo y motor. La tasa de supervivencia fue $98 \%$ para toda la población. La tasa de PC fue similar en los dos grupos, 23/352 en expuestos y en 30/336 no expuestos (OR: 0,70; IC95\% 0,41-1,19). Tampoco se observó diferencia en la tasa de disfunción motora sustancial, 18/352 en los expuestos y 22/336 en los no expuestos (OR: 0,78; IC95\% $0,43-1,43)$. Al valorar PC o muerte no se observó diferencias (OR: 0,65; IC95\% 0,42-1,03). La exposición al MgSO4 fue protector contra disfunción motora severa o muerte (OR: 0,62; IC95\% 0,41-0,93), observándose una reducción del 38\%. 
También en 2007, se publicaron los resultados de una evaluación neonatal después de 18 meses de nacidos, practicada a hijos de mujeres que hicieron parte del estudio (MAGPIE) Multinational Magnesium Sulphate for Prevention of Eclampsia Trial (16), realizado en 129 centros de 19 países en África, Asia, América, Australia y Europa. El estudio había concluido años antes que el MgSO4 es efectivo para la prevención de convulsiones en gestantes con preeclampsia. Fueron analizados 1635 nacidos de madres que recibieron MgSo4 y 1648 niños de madres que recibieron placebo, con edad gestacional menor a 37 semanas para establecer discapacidad neurosensorial o muerte. La tasa 245/1635 $(15,0 \%)$ de los expuestos presentaron muerte o discapacidad neurosensorial no congénita comparada con $233 / 1648(14,1 \%)$ de los no expuestos (RR: 1,06; IC95\% 0,90-1,25). Tampoco hubo diferencia en el riesgo de discapacidad neurosensorial a los 18 meses de nacidos (RR: 1,07; IC95\% 0,92-1,24). El riesgo relativo de mortalidad (RR: 1,11; IC95\% 0,93-1,31), PC (RR: 0,40; IC95\% 0,08-2,05) y disfunción motora sustancial (RR: 2,99; IC95\% 0,12$73,3)$, fue similar y sin diferencia significativa.

Al siguiente año, se publicaron los resultados del estudio multicéntrico Beneficial Effects of Antenatal Magnesium Sulfate (BEAM) (17); 2241 mujeres con parto inminente entre 24 y 31 semanas fueron randomizadas a MgSO4 o placebo. El riesgo de muerte fue similar $9,5 \%$ en expuestos y $8,5 \%$ en no expuestos (RR: 1,12; IC95\% 0,85-1,47). PC moderada/severa fue menos frecuente en el grupo de MgSO4 que en grupo placebo, 1,9\% vs 3,5\% (RR: 0,55 ; IC95\% 0,32-0,95), señalando el papel protector neuronal prenatal del medicamento.

La Tabla I presenta los regímenes de tratamien- to utilizados en cada uno de los cinco ensayos clínicos señalados.

Ninguno de los ensayos clínicos publicados presentaba resultados concluyentes de la importancia del MgSO4 para protección neuronal prenatal. En el año 2009 se publicó el metaanálisis de Costantine y Weiner (19), que incluyó los cinco estudios señalados y 5235 NP de menos de 32-34 semanas de gestación fueron considerados para la revisión. La exposición a MgSO4 se asoció a reducción del riesgo de muerte o PC moderada/severa (RR: 0,85; IC95\% 0,78-0,99), PC de cualquier severidad (RR: 0,70; IC95\% 0,55-0,89), PC moderada/severa (RR: 0,60; IC95\% 0,43-0,84). No se encontró significancia cuando se valoró muerte o PC. En el subgrupo de 3107 nacidos antes de 30 semanas los resultados fueron similares. Muerte o PC moderada/severa (RR: 0,84; IC95\% 0,71-0,99), PC de cualquier severidad (RR: 0,69; IC95\% 0,52-0,92), PC moderada/severa (RR: 0,54; IC95\% 0,36-0,80). Los resultados confirmaban los hallazgos del BEAM y los autores señalaban que la exposición al MgSO4 en mujeres en riesgo de PP reducía $\mathrm{PC}$ en el infante sin incrementar el riesgo de muerte.

También en 2009 Conde-Agudelo y Romero (20), publicaron otro metaanálisis para determinar si MgSO4 en mujeres en riesgo de PP antes de las 34 semanas de gestación reducía la prevalencia de PC. Analizaron de los cinco ensayos clínicos señalados, 4796 mujeres y 5357 niños. Observaron reducción en el riesgo de PC (RR: 0,69; IC95\% 0,55-0,88), PC moderada/severa (RR: 0,64; IC95\% $0,44-0,92)$ y disfunción motora sustancial (RR: 0,60 ; IC95\% 0,43-0,83), sin diferencia en el riesgo de mortalidad pediátrica (RR: 1,01; IC95 0,89-1,14). Ellos concluyen que la administración de MgSO4

Tabla I

RÉGIMEN DE ADMINISTRACIÓN PRENATAL DEL MgSO4

\begin{tabular}{llll}
\hline ESTUDIOS & RÉGIMEN & DURACIÓN & RETRATAMIENTO \\
\hline $\begin{array}{l}\text { MagNET } \\
2002\end{array}$ & $\begin{array}{l}\text { Inicial } 4 \text { gramos } \\
\text { Mantenimiento 2-3 gramos/hora }\end{array}$ & No reportado & No reportado \\
ACTOMgSO4 & Inicial 4 gramos en 20 minutos & Hasta el parto o \\
2003 & Mantenimiento 1 gramo/hora & por 24 horas & No aplicado \\
PREMAG & Inicial 4 gramos & 30 minutos & No aplicado \\
2007 & & & \\
MAGPIE & Inicial 4 gramos en 15 minutos & Hasta el parto o & No aplicado \\
2007 & Mantenimiento 1 gramo/hora & por 24 horas & \\
BEAM & Inicial 6 gramos en 200 minutos & Hasta el parto o & Si, riesgo inminente de PP \\
2008 & Mantenimiento 2 gramos/hora & & $\begin{array}{l}\text { nuevamente, después de } \\
\text { más de } 6 \text { horas de retirado el } \\
\text { MgSO4 }\end{array}$ \\
\hline
\end{tabular}


en mujeres en riesgo de PP antes de 34 semanas reduce el riesgo de $\mathrm{PC}$.

Colaboración Cochrane publicó en ese mismo año otro metaanálisis (21) y una revisión en el año 2010, realizado con los cinco ensayos clínicos ya señalados. En 6145 niños NP, se encontró que la administración de MgSO4 a mujeres con riesgo de PP inminente se redujo de manera significativa el riesgo de PC (RR: 0,68; IC95\% 0,54-0,87). También reducción significativa de la PC moderada/severa (RR: 0,64; IC95\% 0,44-0,92). El riesgo absoluto de PC fue del $3,7 \%$ en expuestos y del $5,4 \%$ en no expuestos, para una reducción absoluta del riesgo del $1,7 \%$. Se observó reducción en PC en nacidos de menos de 34 semanas (RR: 0,69; IC95\% 0,54-0,88) en los cinco estudios, incluyendo 5357 niños. También se encontró reducción significativa en la tasa de disfunción motora gruesa sustancial en 5980 NP de cuatro de los ensayos clínicos (RR: 0,61; IC95\% $0,44-0,85)$. No se observó efectos estadísticamente significativos del tratamiento prenatal con $\mathrm{MgSO} 4$ sobre la mortalidad pediátrica en los cinco ensayos clínicos (RR: 1,04; IC95\% 0,92-1,17). Tampoco sobre otras discapacidades neurológicas en el primer año de edad. Los autores concluyeron que se había establecido el papel protector neuronal prenatal del MgSO4 en NP.

La Tabla II presenta condensados los principales resultados de los cinco ensayos clínicos y los tres metaanálisis considerados.

Mecanismos de protección neuronal prenatal del MgSO4. Existen propuestas teóricas que buscan explicar los potenciales mecanismos de la protección neuronal prenatal que ofrece el MgSO4
(22), las cuales han sido señaladas por Costantine y Drever (23), así como por Marret et al (8).

1. Estabilidad hemodinámica. El MgSO4 parece realizar efectos benéficos sobre la presión arterial, efecto vasodilatador en arterias cerebrales, incrementa el flujo sanguíneo cerebral y minimiza la hipoxia y el daño inducido por isquemia $(8,24)$.

2. Estabilización neuronal. Al prevenir o disminuir el estímulo excitatorio. En la fase aguda después de la lesión, la falta de oxígeno activa el metabolismo anaerobio lo que impide las funciones celulares, se disminuye el Adenosin Trifosfato (ATP) y se acumula ácido láctico. Esto conlleva acumulación de sodio, calcio, cloro y agua, estado denominado edema citotóxico. Neurotransmisores excitatorios como el glutamato, contribuyen al incremento del calcio y el sodio en las neuronas post-sináptica. El MgSO4 bloquea los receptores de N-metil-D-aspartato (NMDA) al glutamato e inhiben la entrada de calcio a las células, atenuando la propagación del potencial de acción. El MgSO4 tiene efectos protectores en modelos animales de daño cerebral $(8,25)$.

3. Propiedades antioxidantes. El aumento del calcio intracelular por lesión isquémica inhibe la activación de las lipasas, proteasas, endonucleasas y fosfolipasas, dando lugar a daño neuronal y cerebral irreversible. Se generan sustancias con efectos citotóxicos: ácidos grasos libres, radicales de oxígeno, óxido nítrico, los cuales superan los mecanismos antioxidantes. En modelos animales se ha comprobado que el $\mathrm{MgSO} 4$ reduce este tipo de reacción $(8,26)$.

4. Propiedades anti-inflamatorias. Posterior a la hipoxia se liberan mediadores inflamatorios (IL-1,

Tabla II

PROTECCIÓN NEURONAL PRENATAL DEL MgSO4

\begin{tabular}{llllll}
\hline Estudios & $\begin{array}{l}\text { Semanas } \\
\text { de edad } \\
\text { gestacional }\end{array}$ & Parálisis cerebral & $\begin{array}{l}\text { Ra (IC95\%) } \\
\text { Parálisis cerebral } \\
\text { moderada/ severa }\end{array}$ & $\begin{array}{l}\text { Muerte o dis- } \\
\text { función motora } \\
\text { sustancial }\end{array}$ & Muerte \\
\hline ACTOMgSO4 $\left(^{*}\right)$ & $<30$ & $0,83(0,54-1,27)$ & $0,53(0,30-0,93)$ & $0,75(0,59-0,96)$ & $0,83(0,64-1,09)$ \\
PREMAG $\left(^{*}\right)$ & $<33$ & $0,70(0,41-1,19)$ & $0,78(0,43-1,43)$ & $0,62(0,41-0,93)^{1}$ & $0,85(0,55-1,32)^{1}$ \\
MAGnet $\left(^{*}\right)$ & $25-33+6$ & $0,94(0,20-4,53)$ & No informado & No informado & $1,93(0,19-20,18)$ \\
BEAM $\left(^{*}\right)$ & $24-31+6$ & $0,59(0,40-0,85)$ & $0,56(0,33-0,95)$ & No informado & $1,12(0,85-1,47)$ \\
MAGPIE $\left(^{*}\right)$ & $25-33+6$ & $0,66(0,11-3,94)$ & No informado & $1,11(0,94-1,32)$ & $1,11(0,93-1,32)$ \\
COSTANTINE $\left(^{* *}\right)$ & $<32-34$ & $0,70(0,55-0,89)$ & $0,60(0,43-0,84)$ & No informado & $1,01(0,89-1,14)$ \\
COSTANTINE $\left(^{* *}\right)$ & $<30$ & $0,69(0,52-0,92)$ & $0,54(0,36-0,80)$ & No informado & $1,00(0,87-1,15)$ \\
CONDE-AGUDELO(**) & $<34$ & $0,69(0,55-0,88)$ & $0,64(0,44-0,92)$ & $0,60(0,43-0,88)$ & $1,01(0,89-1,14)$ \\
COCHRANE $\left(^{* *}\right)$ & $<34$ & $0,69(0,54-0,87)$ & $0,64(0,44-0,92)$ & $0,61(0,44-0,85)$ & $1,04(0,92-1,17)$ \\
\hline
\end{tabular}

$\left(^{*}\right)$ Ensayo Clínico. $\left({ }^{* *}\right)$ Metaanálisis. $\left({ }^{1}\right)$ Odds Ratio (OR). 
FNT). Se ha mostrado que el MgSO4 disminuye estas citoquinas proinflamatorias $(8,27)$.

Costantine y Drever (23) señalan que si bien todos esos cambios han sido observados, el mecanismo exacto por el cual el MgSO4 administrado de forma prenatal protege contra la PC en NP, no es conocido.

Recomendaciones y protocolos vigentes. En 2010 el Colegio Americano de Obstetricia y Ginecología (28) reconoció que la evidencia disponible sugería que el MgSO4 administrado antes del PP reducía el riesgo de PC en los infantes supervivientes, sin establecer punto de corte. Recomienda a los médicos usar MgS04 de forma prenatal para protección neuronal siguiendo los criterios de inclusión (24-31 semanas gestacionales y PP inminente), régimen de tratamiento (6 gramos iniciales más infusión de 2 gramos por hora) y duración (hasta el parto o doce horas) con los cuales se realizó el estudio BEAM (17).

La Sociedad de Obstetricia y Ginecología de Canadá, publicó en 2011 la guía para la práctica clínica (29) donde recomendaba para mujeres con PP inminente ( $\leq 31$ semanas +6 días) la administración de MgSO4 para protección neuronal fetal y consideraba que existía la mejor calidad de la evidencia y el más alto grado para justificar la recomendación (IA). También señalaba: si el MgSO4 ha sido iniciado para protección neuronal, la tocolisis debe ser discontinuada (III-A). EI MgSO4 debía ser discontinuado si el PP no se produjo o administrarse máximo por 24 horas (II-2B). También señaló que era insuficiente la evidencia para repetir la administración prenatal del MgSO4 (IIIL) y la dosis a administrar era 4 gramos como dosis inicial en treinta minutos, seguido de una infusión de 1 gramo por hora hasta 24 horas o el nacimiento (II-2 B). Un año antes se había publicado una guía Australiana (30) donde se señalaba que existía excelente evidencia, que el MgSO4 ofrecía protección neuronal prenatal, recomendaban iguales dosis que las señaladas por los canadienses, más limitaban la recomendación a menos de 30 semanas de gestación, ya que consideraron ser más claro el beneficio y desearon ser prudentes ante el impacto que la aplicación clínica tuviese en la asignación de los recursos (29).

El punto de corte de la edad gestacional para considerar administrar MgSO4 es controversial, por los aplicados en los diferentes ensayos clínicos (22) y en las recomendaciones $(29,30)$. Se ha señalado como límite superior el rango 30-34 semanas de gestación. No obstante, en uno de los metaanálisis (19) el riesgo relativo de PC moderada/severa, muerte o PC moderada/severa y solo PC, fue significativamente favorable tanto para NP antes de 32-34 semanas como para los nacidos antes de las 30 semanas. Con la reducción similar del riesgo de PC en dos grupos se pudiese llevar el punto de corte a las 34 semanas de gestación. El metaanálisis Cochrane (21) también puede soportar la decisión ya que encontraron entre menores de 34 semanas de edad gestacional, PC en 104/2658 expuestos y en 152/2699 de los no expuestos (RR: $0,69 ; 0,54-0,88)$. No obstante el consenso Canadiense (29) decidió dejar las 32 semanas como el límite adecuado, señalan entre otras razones que la administración de MgSO4 a edades cercanas a las 34 semanas de gestación conllevaría incremento y potencial abuso del medicamento, cuando a esas edades existe menor probabilidad de morbilidad neurológica.

No existe consenso en la dosis y esquema de administración. Los ensayos clínicos fueron realizados con distintas dosis de inicio (4 o 6 gramos) y de mantenimiento (ninguna, infusión a 1 gramo o 2 gramos por hora). Una reducción significativa del $41 \%$ del riesgo de PC fue observada en el BEAM (17), estudio que utilizó el régimen condosis más alta (6 gramos al inicio, más infusión de 2 gramos por hora). Esa dosis es recomendada en un protocolo (31). La guía canadiense (29) recomienda 4 gramos iniciales y un gramo por hora. Se ha señalado (32) que no existe diferencia en el efecto dosisrespuesta entre diversos esquemas y la tendencia actual es recomendar el uso de régimen de bajas dosis (4 gramos más 1 gramo por hora) debido a que tiene mejor perfil de seguridad materno-fetal y mayor utilización en la práctica clínica, siendo similar al utilizado como profilaxis-tratamiento de la eclampsia.

En los estudios ACTOMgSO4 (13) y MAGPIE (16) se administró la infusión por máximo de 24 horas, y así lo recomienda la Sociedad de Obstetricia y Ginecología del Canadá (29). Otros autores (31) limitan su uso a 12 horas, por el esquema utilizado en el estudio BEAM (17). Para mujeres con PP programado por indicación materna o fetal, la Sociedad de Obstetricia y Ginecología del Canadá (29) recomienda iniciar MgSO4 antes de cuatro horas del parto con un nivel de evidencia II-2 y grado de recomendación $\mathrm{B}$. Es conocido que el MgSO4 cruza rápida y activamente la placenta, en humanos se alcanzan niveles séricos fetales a la hora y en líquido amniótico luego de 3 horas de administración intravenosa detectables. La vida media del MgSO4 es de 6 horas $(8,22)$.

El beneficio de un nuevo tratamiento en pacientes quienes completaron un curso inicial de MgSO4 está pobremente documentado. Solo el estudio BEAM (17) consideró retratamiento. Estos investigadores administraron de nuevo altas dosis de MgSO4 si el riesgo de PP fue considerado nuevamente inminente y la infusión inicial había sido discontinuada por más de 6 horas. El protocolo y el algoritmo propuesto por Reeves y cols (31), lo recomiendan si la paciente tiene de nuevo riesgo inmi- 
nente de PP. Si han transcurrido más de seis horas de finalizado el esquema previo se coloca 6 gramos en 30 minutos y se sigue a 2 gramos hora por doce horas. Si no han transcurrido las seis horas de suspensión se coloca la infusión de 2 gramos horas por doce horas. Otros (29), consideran que la evidencia es insuficiente para recomendar la repetición del MgSO4 con fines de protección neuronal prenatal.

La administración del MgSO4 se debe acompañar de los cuidados maternos y fetales, tomando en consideración los efectos secundarios y los riesgos de toxicidad. Son contraindicaciones fetales la muerte intrauterina, las malformaciones congénitas letales; y maternas la miastenia gravis, la cardiopatía grave, el bloqueo cardiaco, la insuficiencia respiratoria, las alteraciones hidroelectrolíticas con hipocalemia o hipocalcemia y la insuficiencia renal (31). Números de partos pretérminos necesarios a tratar. No existe tratamiento curativo para la PC, por tanto las medidas preventivas son de gran importancia. El beneficios del MgSO4 como estrategia para reducir el riesgo de PC en NP se puede estimar según el número necesario a tratar (NNT, number needed to treat) para la prevención de un caso de PC.

El metaanálisis de Costantine y Weiner (19), ha calculado en 56 [IC95\% 34-164] el NNT con MgSO4 antes de las 32-34 semanas de gestación para prevenir 1 caso de PC hasta los 18-24 meses de nacido, y en 46 [IC95\% 26-187] el NNT de menos de 30 semanas de gestación para igual prevención.

El metaanálisis de Cochrane (21) señala NNT de 63 [IC95\% 44-155] para prevenir un caso de PC. En el grupo de menos de 34 semanas de gestación y en el de menos de 30 semanas de gestación el NNT para prevención de un caso de PC es 56 y 46, respectivamente.

El metaanálisis de Conde-Agudelo y Romero (20) señala que si todas las mujeres que tuviesen parto antes de las 34 semanas de gestación recibieran MgSO4, el número de nuevos casos de PC que potencialmente se pudiesen prevenir anualmente serían 620 [IC95\% 240-900), cifra importante si se tiene en cuenta que se ha estimado que 8000 neonatos y niños que cada año son diagnosticados de PC en los Estados Unidos (33) y cerca del $25 \%$, o sea 2000 nacen antes de las 34 semanas de gestación (4). Un metaanálisis (34) señaló que la prevalencia de PC disminuye significativamente con el incremento en la edad gestacional (14,6\%; IC95\% 12,5-17,0) en NP extremos (<28 semanas). La PC en nacidos a las 27 semanas es significativamente menor que la sucedida en nacidos con 24,25 y 26 semanas de gestación $(p<0,05)$. No se observó diferencia entre los nacidos a 24, 25 y 26 semanas $(p=0,8)$. En muy pretérmino (28-31 semanas) $(6,2 \%$; IC95\% 4,9-7,8), en moderadamente pretérmino $(0,7 \%$; IC95\% 0,6-0,9) y para nacidos de término (37 y más semanas) $(0,1 \%$; IC $95 \%$ $0,09-0,014)$. Datos similares señala la cohorte de
Noruega (4), donde encontraron PC en el $9,1 \%$ de los nacidos entre $23-27$ semanas, $6,0 \%$ entre $28-30$ semanas, $1,9 \%$ entre $31-33$ semanas, $0,3 \%$ entre 34-36 semanas y $0,1 \%$ de 37 semanas en adelante $(p<0,001)$. Por ello es diferente el NNT para cada edad gestacional.

Conde-Agudelo y Romero (20), también señalan que el NNT es 52 [IC95\% 31-154] mujeres en riesgo de PP antes de 34 semanas para realizar prevención de un caso de PC. Dichos autores toman en consideración un estudio realizado en Estados Unidos que estimó para el año 2005 el costo total de administrar MgSO4 fue US\$197,90 por paciente y propone que si todas las mujeres en riesgo de PP antes de 34 semanas, recibieran MgSO4, el costo de prevenir un caso de PC serían US\$10.281, lo cual es significativamente más costo-efectivo que tener que tratar un caso de PC. Como ya fue señalado, el costo estimado para esos años de la PC fue de un millón de dólares por cada caso (6).

\section{CONCLUSIONES}

La administración de MgSO4 es una estrategia terapéutica prenatal que se puede considerar como un progreso en la intención de reducir la prevalencia de PC en NP. Existe adecuado nivel de evidencia que permite considerar al MgSO4 como protector neuronal prenatal al reducir significativamente la PC, en PP inminente o PP programado. Si bien existen controversias en la dosis y en la edad gestacional de punto de corte, tal vez no se tengan justificaciones para no considerarlo en la práctica clínica, teniendo en cuenta la elevada prevalencia de PP a nivel mundial y los altos costos asociados a la PC.

\section{REFERENCIAS}

1. Garite TJ, Combs CA. Obstetric interventions beneficial to prematurely delivering newborn babies: antenatal corticostetroids, progesterone, magnesium sulfate. Clin Perinatol 2012;39:33-45.

2. Engle WA. Morbidity and mortality in late preterm and early term newborns: a continuum. Clin Perinatol 2011;38:493-516.

3. Saigal S, Doyle LW. An overview of mortality and sequelae of preterm birth from infancy to adulthood. Lancet 2008;371:261-9.

4. Moster D, Lie RT, Markestad T. Long-term medical and social consequences of preterm birth. $\mathrm{N}$ Engl $\mathrm{J}$ Med 2008;359:262-73.

5. Yeargin-Allsopp M, Van Naarden Braun K, Doernberg BA, Benedict RE, Kirby RS, Durkin MS. Prevalence of cerebral palsy in 8-year-old children in three areas of the United States in 2002: a multisite collaboration. Pediatrics 2008;121:547-54.

6. Honeycutt A, Dunlap L, Chen H, Housi G. Economic costs associated with mental retardation, cerebral palsy, hearing loss, and vision impairment-United States 2003. MMWR 2004;53:57-9.

7. Hans P, Bonhmme V. Neuroprotection with anaesthetic agents. Curr Opin Anaesthesiol 2001;14:491-6.

8. Marret S, Doyle LW, Crowther CA. The antenatal magnesium sulphate for neuroprotectionin the preterm infant. Sem Fetal Neonat Med 2007;12:311-7. 
9. Leviton A, Kuban KC, Pagano M, Brown ER, Krishnamoorthy KS, Allred EN. Maternal toxemia and neonatal germinal matrix hemorrhage in intubated infants less than 1751g. ObsteGynecol 1988;72:571-6.

10. Van de Bor M, Verloove-Vanhorick SP, Brand R, Keirse MJ, Ruys JH. Incidence and prediction of periventricular-intraventricular hemorrhage in very preterm infants. J Perinat Med 1987;15:333-9.

11. Nelson KB, Grether JK. Can magnesium sulfate reduce the risk of cerebral palsy in very low birthweight infants? Pediatrics 1995;95:263-9.

12. Mittendorf R, Dambrosia J, Pryde P, Lee K-S, Gianopoulos $\mathrm{J}$, Besinger $\mathrm{R}$, et al. Association between the use of antenatal magnesium sulfate in preterm labor and adverse health outcomes in infants. Am J ObstetGynecol 2002;186:1111-8.

13. Crowther CA, Hiller JE, Doyle LW, Haslam RR. Australasian Collaborative Trial of Magnesium Sulphate (ACTOMgSO4) Collaborative Group. Effect of magnesium sulfate given for neuroprotection before preterm birth. $A$ randomized controlled trial. JAMA 2003:290:2669-76.

14. Marret S, Marpeau L, Zupan-Simunek V, Eurin D, Leveque $C$, Hellot MF, Benichou J. Magnesium sulphate given before very-preterm birth to protect infant brain: the randomized controlled PREMAG trial. BJOG 2007;114:310-8.

15. Marret S, Marpeau L, Follet-Bouhamed C, Cambonie G, Astruc D, Delaporte B, et al. [Effetc of magnesium sulphate on mortalily and neurologic morbidity of the very-preterm newborn (of less than 33 weeks) with two-year neurological outcome: results of the prospective PREMAG trial]. [Article in French]. Gynecol Obstet Fertil 2008;36:278-88.

16. Magpie Trial Follow-Up Study Collaborative Group. The Magpie trial: a randomized trial comparing magnesium sulphate with placebo for pre-eclampsia. Outcome for children at 18 months. BJOG 2007;114:289-99.

17. Rouse DJ, Hirtz DG, Thom E, Varner M, Spong C, Mercer $\mathrm{B}$, et al. A randomized, controlled trial of magnesium sulfate for the prevention of cerebral palsy. $\mathrm{N}$ Engl J Med 2008;359:895-905.

18. Ancel PY, Marret S, Larroque B, Arnaud C, ZupanSimunek V, Voyer M, et al. Are small for gestational age and maternal hypertension risk factors for severe intraventricular hemorrhage and cystic periventricular leucomalacia compared with other causes of very preterm birth? Results of the EPIPAGE cohort study. Am J ObstetGynecol 2005;193:178-84.

19. Costantine MM, Weiner SJ. Effects of antenatal exposure to magnesium sulfate on neuroprotection and mortality in preterm infants. Obstet Gynecol 2009;114:354-64.

20. Conde-Agudelo A, Romero R. Antenatal magnesium sulfate for the prevention of cerebral palsy in preterm infants less than 34 weeks' gestation: a systematic review and metaanalysis. Am J Obstet Gynecol 2009;200:595-609.
21. Doyle LW, Crowther CA, Middleton P, Marret S, Rouse M, Magnesium sulphate for women at risk of preterm birth for neuroprotection of the fetus. Cochrane Database Syst Rev 2009;1:CD004661.

22. Heyborne KD. Magnesium sulfate neuroprotection:time to start? Postgraduate Obstet Gynecol 2010;30:1-8.

23. Costantine MM, Drever N. Antenatal exposure to magnesium sulfate and neuroprotection in preterm infants. Obstet Gynecol Clin N Am 2011;38:351-66.

24. Keeley MM, Wade RV, LaurentSL, Hamann VD. Alterations in maternal-fetal Doppler flow velocity waveforms in preterm labor patients undergoing magnesium sulfate tocolysis. Obstet Gynecol 1993;81:191-4

25. Marret S, Gressens P, Gadisseux JF, Evrard P. Prevention by magnesium of excitotoxic neuronal death in the developing brain: an animal model for clinical intervention studies. Dev Med Child Neurol 1995;37:473-84.

26. Spandou E, Soubasi V, Papoutsopoulou S, et al. Neuroprotective effect of longterm MgSO4 administration after cerebral hypoxia-ischemia in newborn rats is related to the severity of brain damage. Reprod Sci 2007; 14:667-77.

27. Galvin KA, Oorschot DE. Postinjury magnesium sulfate treatment is not markedly neuroprotective for striatal medium spiny neurons after perinatal hypoxia/ ischemia in the rat. Pediatr Res 1998;44:740-5.

28. American College of Obstetricians and Gynecologists Committee on Obstetric Practice, Society for Maternal-Fetal Medicine. Magnesium sulfate before anticipated preterm birth for neuroprotection. Committee Opinion No. 455: Obstet Gynecol 2010;115:669-71.

29. Magee L, Sawchuck D, Synnes A, Von Dadelszen P. SOGC Clinical Practice Guideline. Magnesium sulphate for fetal neuroprotection. J Obstet Gynaecol Can 2011;33:516-29.

30. The Antenatal Magnesium Sulphate for Neuroprotection Guideline Development Panel. Antenatal magnesium sulphate prior to preterm birth for neuroprotection of the fetus, infant and child: National clinical practice guidelines. The Australian Research Center for Health of Women and Babies, The University of Adelaide, 2010

31. Reeves SA, Gibbs RS, Clark SL. Magnesium for fetal neuroprotection. Am J Obstet Gynecol 2011;204:202. e1-4.

32. Cahill A, Caughey A. Magnesium for neuroprophylaxis: fact or fiction? Am J Obstet Gynecology 2009;200:590-4.

33. United Cerebral Palsy. Press Room. Vocabulary tips. Cerebral Palsy-Facts \& Figures. Disponible en: http:// www.ucp.org/ucp_generaldoc.cfm/1/9/37/37/447. Acceso 20 de enero de 2009.

34. Himpens E, Van den Broeck C, Oostra A, Calders P, Vanhaesebrouck P. Prevalence, type, distribution, and severity of cerebral palsy in relation to gestational age: a meta-analytic review. Dev Med Child Neurol 2008;50:334-40. 